\title{
The Protective Effect of Scientific Vocal Training on Voice
}

\author{
Tian Lei, Qu Yan \\ Heilongjiang University of Technology, Heilongjiang, China
}

Keywords: Voice Training; Scientific Voice Production; Voice Protection

Abstract: In daily voice training, it is necessary to use scientific methods in carrying out training step by step, which can not only meet the requirements of professional vocal music training, but also achieve the effect of voice protection in the process of training according to scientific methods. In the practical application of the principle of scientific occurrence, first of all, it is necessary to carry out adaptive and targeted training on the premise of each trainer's own voice characteristics. This process is not only the premise and basis for learning scientific voice, but also the basis conditions for further training planning. Because there are differences in pronunciation conditions, voice mastery skills and comprehension abilities among different people, how to formulate different training strategies in training, and how to adopt different training methods and intensity for different trainers through scientific methods are important criteria to measure whether the final training objectives can be achieved.

\section{Introduction}

The structure of human body has certain regularity and particularity, so does the vocal system. In fact, the art of vocal music singing is the combination of natural voice, scientific vocal method and music. Therefore, special attention should be paid to the scientific use and protection of voice in the process of vocal music training. The famous Italian legendary tenor Caruso has won countless praises and honours in his legendary life. The number of times he has sung in his 25 years of professional singing career is amazing. The number of opera theatres in New York Metropolitan City alone has reached 863. Because of his frequent use of his voice, he cannot get proper rest, and most importantly, he is an addict. There are relevant data to prove that long-term excessive smoking is also one of the culprits of his premature death. He has a great influence on many singers of the same era and later generations. His beautiful and graceful voice and the eventual early death of illness also give the singers of later generation space to study and summarize, so that we can conduct more scientific research on human voice system more seriously and rationally. In today's vocal music training, we need to combine more scientific research results to examine the voice training and protection, and ultimately apply them to actual training.

\section{The Importance of voice protection and psychological adjustment}

In the current training of vocal music singing, unscientific training content and overload training 
plan of trainers often lead to vocal cord damage. This is because in the professional vocal music performance process, the singing requirements of actors are more stringent, which usually need to repeat the training in a single tone or individual articulation. This series of training processes will undoubtedly be repeated many times in a short time, the voice parts and the associated muscles are the same. The purpose is to mobilize the whole voice system for scientific pronunciation. In daily life, when using the vocal system in normal state, there is seldom such a voice state requiring the vocal producer, so the intensity of the vocal cords used by the generating system is often difficult to accept. If the training method is not scientific and reasonable, the above-mentioned vocal cord damage will occur.

In the case of vocal cord damage, vocal music trainers not only cannot carry out normal training, but also will inevitably lead to the decline of singing function and level, more terrible is that many times it will also cause a certain psychological burden. Therefore, the importance of voice protection is beyond doubt. It requires all the personnel involved in the singing training to pay enough attention to the voice protection. It is absolutely not only that the instructor of this training should make a scientific plan for the trainees in the actual teaching, but also that the vocal music training should be carried out step by step according to the scientific plan in the course of training. Never be eager to achieve something and be ambitious, but turn the tables on the contrary and discard all the previous achievements.

\section{The effect of vocalization on voice health}

\subsection{The impact of pursuing voice quality}

Not everyone has a beautiful voice. For people with better congenital conditions, a little training can basically meet the requirements. But for many people, there will inevitably be some defects in timbre. For example, some people's voices are not bright enough, some are not smooth enough, some are not generous enough, some are not concentrated enough, and so on. If there is no scientific method of voice production, many people have to squeeze and squeeze their throats to obtain the required voice quality. In the long run, such voice production can easily cause voice damage. Some people pursue a personalized timbre, thinking that the voice of emptiness is a magnetic voice. In fact, speaking like this for a long time may lead to the risk of losing the ability to invent bright and real sounds. At the same time, the inability to correctly grasp the empty voice will inevitably affect the clarity of expression.

\subsection{The impact of voice modelling composition}

Voice modelling refers to the shaping of characters through their own voice and voice. Different characters need different voice shapes, because different roles have different personality characteristics, different levels of education, different growth and living environment, and different voice patterns, which is consistent with the situation of people in life. In addition, different age groups of the same role, its timbre is also significantly different. It's a big test how to make the voice that the character needs without hurting his voice. In the application, if the method is not appropriate, it may also bring trouble to the voice.

\subsection{The impact of pursuing the voice penetration}

Sound penetration comes from loudness, height, brightness and intensity. Such voice is not the voice used in life, it needs long-term training. First of all, the loudness of the sound needs strong voice power, that is to say, it needs sufficient breath support, and the density of the breath is large. 
The loudness of sound needs to increase the amplitude of vocal cord vibration. The loudness of sound also needs plenty of resonance, so the resonant cavity is smoother than the living language, and the space of the resonant cavity should be expanded appropriately. Secondly, the height of the sound needs to increase the density of the breath to expand the subglottic pressure and the speed of the vocal cord vibration. Finally, the strength of voice is positively related to the strength of breath, the strength of voicing, and the tension of the vocal organs. However, when the voice is loud and loud, the intensity of pronunciation should be moderately weakened, otherwise it will be harsh. On the contrary, when the voice is weak and low, the intensity of pronunciation must be increased. For the penetration of sound, if there are no good vocal skills, it may become hoarse and exhaustive shouting, the vocal organs may bear tremendous pressure, and damage is inevitable.

\subsection{The impact of persistent vocalization}

High-intensity voice, voice fatigue easily. Once the voice is tired, the ability of cooperation among organs in the vocal system will be greatly reduced, and the ability of movement of each organ itself will also be reduced, and the quality of sound may not be guaranteed. If we insist on high-intensity voice under fatigue, acute voice disease will occur, which may lead to chronic voice disease in the long run. The endurance of vocalization varies from person to person. Those who are physically strong, breathe and sound smooth, have a relaxed throat, and have good voice placement may have a stronger lasting voice, and vice versa, a weaker voice. Of course, the endurance of vocalization can also be improved through long-term scientific training. However, we should not abuse our voice wantonly because of its long-lasting ability.

\section{Basic concept and application of scientific voice training}

The science of vocalization methods should be based on the level of vocal efficiency, whether it conforms to the law of human voice development, and whether it is harmful to human voice health. It can be seen that the scientific method of voice production is to produce the highest quality voice in line with the occupational needs with the minimum energy consumption. It is in line with the law of human voice development and has no harm to the voice health. This method emphasizes the synergistic movement of the vocal organs, the breath is controlled freely, the intensity is moderate, the depth is appropriate; the sound is reasonable, the breath is balanced; the vocal tract is smooth and the resonance is sufficient; the vocal words are accurate, clear and beautiful, natural; the sound quality is mellow and rich in changes. the voice is penetrating, expressive and lasting.

The main reason why scientific vocalization has a good protective effect on human vocal organs is that it can improve the movement mode of the vocal organs, improve the working efficiency of the vocal organs and reduce the damage of the vocal organs.

\subsection{Definition of scientific voice training}

The scientific definition of vocal training methods is mainly based on whether the ultimate learning efficiency and the ability of training vocalists are scientific. Generally, such definition is a universal standard. In the case of the high frequency of vocal use of the trainees, it is necessary to adjust it appropriately according to the specific conditions of the trainees. In addition, because each trainee's voice may have a special decision in vocal music singing training, it is necessary to practice in a variety of ways during the training process. At the same time, in the process of training with scientific methods, it is necessary to make a comprehensive judgment on whether the specific vocal mode will damage the health of vocal cords. Therefore, the specific concepts of scientific vocal training methods can be summarized as follows: in the normal application of vocal cords for 
actual singing, to achieve the lowest frequency of singing, causing the least damage, and ultimately achieve the best training effect of vocal training methods.

\subsection{The effect of scientific vocal training}

In peacetime training process, under the guidance of scientific vocal training method, the vocal system of trainees can get relatively relaxed and balanced coordinated movement, and will not repeatedly use a single part or part of muscle groups and organs for high-intensity training. When carrying out some difficult training, it is necessary to do a good job of vocal cord vocal protection in advance to adapt to the later training process stage. In the use of breath in vocal training, the trainer will present a soft voice, smooth and stable breath, coherent voice, and the whole vocal process is in a controllable range. At the same time, the most expressive language part in the vocal music singing process can also be clearly and accurately expressed, and make the listener feel the singer's professional expression of the content of the song. If we want to further improve the level and apply the usual training to the stage performance, we need the performers to accurately master the control skills of their own voice system based on the usual scientific voice training methods. Only in this way can we more easily interpret works on the stage through voice.

\subsection{Protection of vocal cords by scientific voice training}

In the whole process of vocalization, the system of human body participating in vocalization includes not only the general visual organs such as mouth and nose, but also the internal organs such as larynx and lung. Especially in the vocal music process training, the trainer usually needs to have a strong ability of sustained vocalization. The stability of breath and the muscle groups related to vocalization need much attention and research. Applying the soft voice method in scientific vocal training can keep the relatively stable vibration of vocal cords in use. Of course, the power source of this process is closely related to the stable breath output. Only in this way can the sound organs finally achieve almost perfect and harmonious tacit coordination.

For hoarseness and inadequate closure of vocal cords in vocal training, the use of tongue-stretching singing can appropriately regulate the muscle function of the closed part of vocal cords. This kind of situation occurs more in the process of soprano vocal part training. When the vowels are opened and tongue-stretching, singing will lead to vocal weakness and hoarseness in a large extent due to inadequate closure of vocal cords. but if this method is still unable to change the voice of this kind of situation, it is recommended to go to the hospital for medical diagnosis of vocal cords, often vocal polyps or vocal nodules and other pathological changes, such as vocal cords, vocal cords not only do not recommend vocal training, but also suggest timely medical treatment, in order to avoid affecting future vocal music learning.

It is also the ultimate goal that all vocal music learners aspire to and pursue that they can scientifically and actively adjust the resonant cavity in vocal training, so that the volume of singing can be expanded and the sound quality is smooth and full. In the general pronunciation process, using unscientific methods to enlarge the volume will usually lead to breaking and other phenomena such as forks, which will affect the timbre and pitch of the voice to a certain extent. On the premise of applying scientific vocal training methods, it is possible to maximize the reasonable movement of the resonant cavity, so as to achieve the effect of smooth flow of all parts of the vocal system with the change of sound volume, and achieve the stability, gracefulness and smoothness of sound quality while enlarging sound. Because singing controls and opens the variable resonant cavity sufficiently, it gains enough channel and space for the smooth transmission of breath and sound, and with good vocal cord closure, it will surely make the resonance more fully. In addition, it is also a very important singing skill that whether the voice can finally reach the so-called mask position in 
the pronunciation, because the voice can stimulate the trigeminal nerve, which makes the voice brighter and improve the penetration of the voice. Scientific pronunciation often requires that the direction of singing and the direction of breath and the state of the whole body are reversed. With the rise of pitch, the center of gravity and breath of the whole body are absolutely unavoidable. The fulcrum of pitch and breath of singing and the fluctuation of the whole body must not be in the same direction. Of course, the mastery of this singing skill must be based on a lot of scientific pronunciation training, as well as a large number of singing experience under the accumulation of continuous exploration and mastery.

\subsection{Protection of vocal cords by connecting the resonant chambers}

The enlargement of sound volume in scientific pronunciation mainly depends on the support of increasing breath and enlarging resonance. To expand the resonance, we must open and expand the variable resonance chamber appropriately, so that the resonance of the lower, middle and upper parts of the voice can change freely with the need of expression. Due to the full opening of the variable resonant chamber, it has won enough channels for the smooth breath and sound, and good glottis closure, which will certainly make the resonance more fully. This not only solves the problem of improving the timbre, but also increases the soundness and penetration of the voice, thus improving the efficiency of voice production and reducing the wear and tear of the voice. Provide enough power to reduce the impact on vocal cords. Scientific vocal breathing is mainly controlled combined breathing. This way of breathing can make the breath inhaled into the lungs bottom, which increase the quantity, depth and density of breath. It can also be fully regulated through the back waist to make the breath abundant, easy to control, and provide enough power for vocalization.

Finally, sound delivery to the mask can improve the penetration of the voice. Thus, scientific voice can not only meet the demand for sound, but also greatly reduce the damage to the voice, so it can also make the voice more durable.

\section{Conclusion}

Vocal music singing art is widely loved by people at present. The number of trainees who are willing to accept professional training is increasing. Therefore, it is necessary to adopt scientific vocal methods in the process of training. Scientific vocalization training can not only meet the needs of vocal music performance for singing, but also greatly reduce the damage to vocal cords, and make vocalization more lasting. Therefore, the fundamental method of treatment is to thoroughly solve the problem of vocal music learners and singers' voice damage, and learn scientific methods of voice production.

\section{Acknowledgement}

Source: Social Science Federation project of Jixi: "Research of the scientific voice training to alleviating the common voice disease” project number: 2018 JSK005.

\section{References}

[1] Zhao Fengyan. Scientific vocalization and voice protection [J]. Huang Zhong (Journal of Wuhan Conservatory of Music), 2003(s1): 93-95.

[2] Lv Wei, Liu Haibing, Wang Junxian. Discussion on the Maintenance of Throat Function in Voice Medicine [J]. Journal of Sichuan Medical Sciences, 2006, 27(3): 310-311.

[3] Zhou Weimin. Scientific Voice and Artistic Voice [J]. Journal of the Central Conservatory of Music, 1990(4): 43-46. 